\title{
巴西木素类天然产物的合成研究进展
}

\author{
汪学全 $a, b$ 刘 卫 ${ }^{a}$ 段素月 $a$ 羊晓东*,b 张洪涁 $*, b$ \\ ( ${ }^{a}$ 红河学院理学院/云南省天然药物与化学生物学重点实验室 蒙自 661199) \\ ( ${ }^{b}$ 云南大学教育部自然资源药物化学重点实验室 昆明 650091)
}

\begin{abstract}
摘要 巴西木素类型天然产物是从中药苏木(Caesalpinia sappan)中分离得到的一类化合物，因其独特的结构和良好的 生物活性而成为合成化学家关注的焦点. 目前，已有许多关于这类天然产物合成的报道. 我们主要对巴西木素类型天 然产物的合成研究进展进行了综述.

关键词 巴西木素; Brazilide A; Brazilein; 合成
\end{abstract}

\section{Research Progress on the Synthesis of Brazilin-Type Natural Products}

\author{
Wang, Xuequan ${ }^{a, b} \quad{\text { Liu, } \mathrm{Wei}^{a}}^{a} \quad$ Duan, Suyue $^{a} \quad$ Yang, Xiaodong $^{*, b} \quad$ Zhang, Hongbin ${ }^{*, b}$ \\ ( ${ }^{a}$ Key Laboratory of Natural Pharmaceutical \& Chemical Biology of Yunnan Province/School of Science, Honghe University, \\ Mengzi 661199) \\ $\left({ }^{b}\right.$ Key Laboratory of Medicinal Chemistry for Natural Resource Ministry of Education, Yunnan University, Kunming 650091)
}

\begin{abstract}
Brazilin-type natural products were isolated from the heartwood of Caesalpinia sappan, a famous traditional Chinese medicine. These compounds have stimulated a great deal of interest on synthetic chemists due to their structural novelty and promising biological activities. Up to now, many methods towards the synthesis of these natural products have been developed. Progress on the synthesis of Brazilin-type natural products are reviewed in this paper.

Keywords brazilin; brazilide A; brazilein; synthesis
\end{abstract}

巴西木素类天然产物主要分离自传统中药苏木, 是 苏木中特有的一类化合物, 从 20 世纪 50 年代开始陆续 被发现, 目前已分离鉴定出的主要有以下几种: $(+)$-Brazilin (1) ${ }^{[1]} 、(-)$-Brazilein (2) ${ }^{[2]} 、(+)$-Brazilide A (3)、(+)-Haematoxylin $(4)^{[3]} 、(+)$-Haematoxylane (5) 和 $(+)$-Brazilane (6) 等 6 个化合物, 其中 $(+)$-Brazilide A (3) 于 2002 年才由中国科学院上海药物研究所叶阳课题组 [4] 首次分离并报道了其结构鉴定工作(图 1).

这类化合物结构具有相似之处, 都具有苯并二氢吡 喃环, 是一类高异黄酮衍生物, 区别主要在于 A 环和 C-3 位的取代. 除了化合物 Brazilein (2)和 Brazilide A (3), 其它化合物的 A 环都是儿茶酚结构, 而 Brazilein (2)是 Brazilin (1) A 环被氧化的产物, Brazilin (1)放置在
空气中或在光照条件下即可部分转化为 Brazilein (2). 因此, Brazilin (1)和 Brazilein (2)在生源途径上主要存在 氧化还原的相互转换历程 ${ }^{[5]}$. 而 Brazilide A (3) 也被认 为是 Brazilin (1) A 环高度的氧化切断而形成双内酯结 构(Scheme 1) $)^{[4]}$.

巴西木素类天然产物是苏木的主要成分, 也是苏木 的主要活性成分, 一直受到广大化学工作者的关注. 其 中, Brazilin 应用非常广泛, 最早被用作天然的红色生物 染色剂 ${ }^{[6]}$, 后续研究进一步发现其具有抗肿瘤 ${ }^{[7]}$ 、降血 糖 $^{[8]}$ 、抗炎 ${ }^{[9]}$ 、抗菌 ${ }^{[10]}$ 、抗血小板 ${ }^{[8]} 、$ 保肝 ${ }^{[11]}$ 等多种生 物活性, 还可作为 DNA 切割剂 ${ }^{[12]}$. 目前 Brazilin 的含量 还是评价药材苏木质量的重要依据, 2010 年版《中国药 典》规定，药材苏木中 Brazilin 的含量不得少于 $0.5 \%{ }^{[13]}$.

*E-mail: zhanghb@ynu.edu.cn, xdyang@ynu.edu.cn

Received March 17, 2015; revised May 3, 2015; published online May 14, 2015.

Project supported by the Program for Changjiang Scholars and Innovative Research Team in University (No. IRT13095), the National Natural Science Foundation of China (Nos. 21462049, 21332007, U1402227), the Natural Science Foundation of Yunnan Province (Nos. 2013FA028, 2012FB113) and the Excellent Young Talents of Yunnan University.

长江学者和创新团队发展计划(No. IRT13095)、国家自然科学基金(Nos. 21462049, 21332007, U1402227)、云南省自然科学基金(Nos. 2013FA028, 2012FB113)、云南大学青年英才培育计划资助项目. 


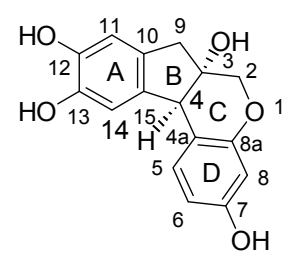

(+)-Brazilin (1)

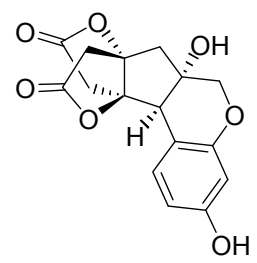

(+)-Brazilide A (3)

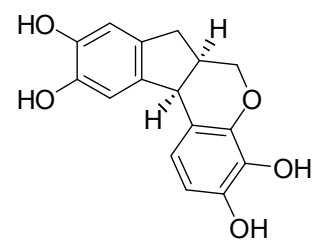

(+)-Haematoxylane (5)

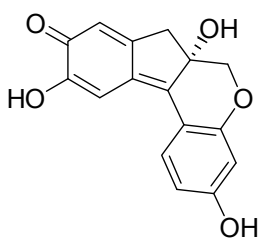

(-)-Brazilein (2)

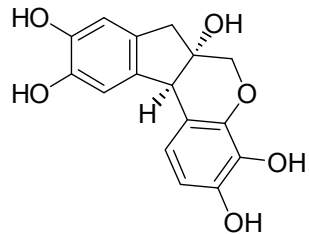

(+)-Haematoxylin (4)

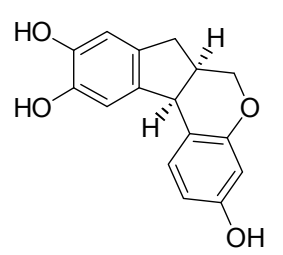

(+)-Brazilane (6)
图 1 巴西木素类天然产物

Figure 1 Brazilin-type nature products

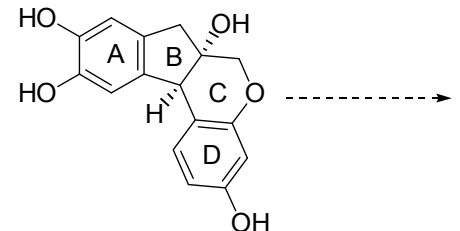

(+)-Brazilin (1)

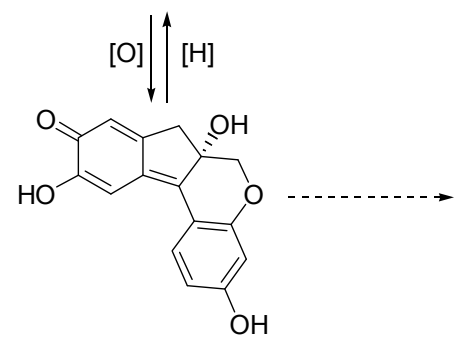

(-)-Brazilein (2)

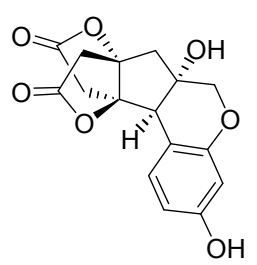

(+)-Brazilide A (3)

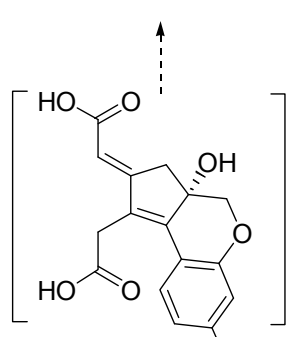

$\mathrm{OH}$
图式 1 Brazilide A 可能的生源合成途径

Scheme 1 Possible biosynthetic pathway for brazilide A

而化合物 Brazilein 结构与 Brazilin 有很多相似之处, 也被证实具有很好的强心作用、免疫抑制活性、抗癌、 神经保护作用等多种活性 ${ }^{[14]}$.

Brazilin 具有多种生物活性, 其结构又代表了巴西 木素类天然产物的基本结构，长期以来是合成工作者非 常重视的一个目标分子. 而 Brazilide A 由于双内酯的独 特结构, 也受到了广大合成工作者的关注, 一度被评为 天然产物的热点分子之一 ${ }^{[15]}$. 由于巴西木素类天然产
物独特的结构和显著的生物活性, 吸引了众多合成化学 家的关注，目前已有许多关于这类天然产物合成的报 道. 本文主要综述了巴西木素类天然产物四环骨架的构 建方法及其全合成研究进展.

\section{1 基于生源途径合成巴西木素类天然产物及其 类似物}

从结构上看, 巴西木素类天然产物属于高异黄酮的 衍生物, 而高异黄酮化合物又广泛存在于苏木的成分 中. 因此, 从生源途径推断, Brazilin 的生物合成前体可 能是高异黄酮化合物 7 (Scheme 2).
图式 2 Brazilin 可能的生物合成前体<smiles>[R20]c1ccc2c(c1)OCC(O)(Cc1ccc([R2])c([R])c1)C2=O</smiles>

早在 1980 年 Heller 和 Tamm 就提出了 Brazilin 在 生物体内可能的合成途径(Scheme 3) ${ }^{[16]}$.

首先 Brazilin 的合成起始于查尔酮化合物 $\mathbf{8}$, 酚差 基氧上的孤对电子转移到碳上，形成羰基，再发生一系 列的电子转移, 最后由羰基 $\alpha$ 位上的碳转移到甲氧基上 形成苯并二氢吡喃环化合物 $\mathbf{1 0}$, 电子转移历程如化合 物 9 所示. 化合物 10 不稳定，电子从羰基 $\alpha$ 位上的碳氢 键转移，发生芳构化形成高异黄酮化合物 11, 而化合物 11 又可以加水形成化合物 7, 化合物 7 脱水同样可以生 成化合物 11. 而化合物 7 可以被还原形成化合物 12、13、 14 和 16. 化合物 12、13、14 又都可以通过氧化去芳化 使 3 '位上的酚羟基氧上孤对电子发生转移, 最后电子转 移到对位碳上，从而进攻苠位的烷氧基碳，碳氧键断裂 形成化合物 15, 化合物 15 脱氢芳构化就形成 Brazilin (1). 1986 年 Nohara 研究组 ${ }^{[17]}$ 从苏木中分离到化合物 7、 11、12、13、14、16 六个天然产物, 发现化合物 12、13、 14 在加热到 $70{ }^{\circ} \mathrm{C}$ 均可以转换为 Brazilin, 进一步佐证 了 Heller 和 Tamm 提出的 Brazilin 在生物体内的合成途 径.

因此，最早关于巴西木素类天然产物的合成研究， 都是基于生源途径来完成的，首先都是要合成前体化合 物 7. 最早可追溯到 20 世纪 70 年代 Kirkiacharian 研究 组 ${ }^{[18]}$ 利用生源合成途径首次报道了 Brazilin 三甲醚化衍 生物的合成(Scheme 4). 


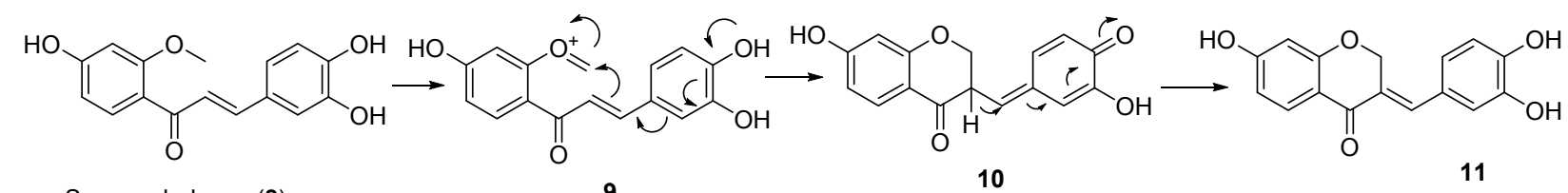

Sappanchalcone (8)

9

10

11

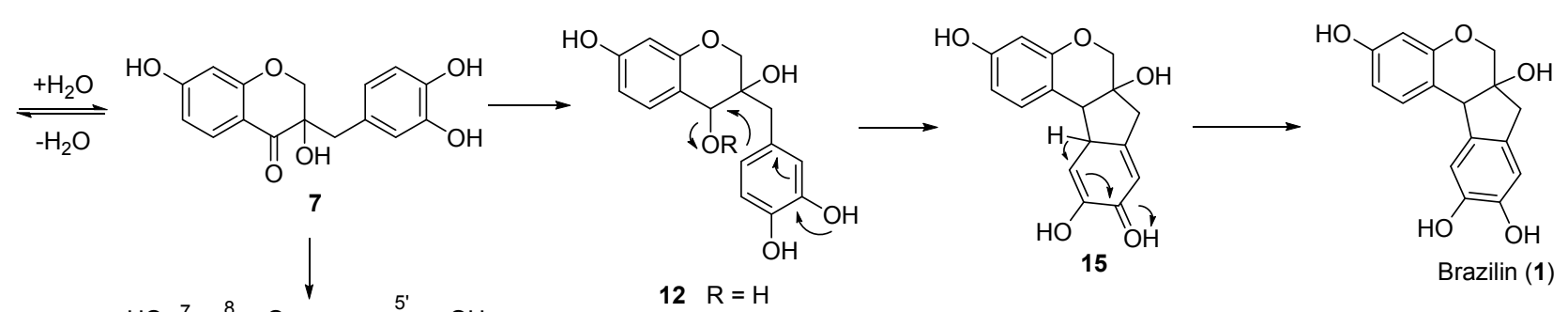

图式 3 Brazilin 可能的生源合成途径

Scheme 3 Possible biosynthetic pathway for brazilin<smiles>COc1ccc(C(=O)CCc2ccc(OC)c(OC)c2)c(O)c1</smiles>

19<smiles>COc1ccc2c(c1)OCC(O)(Cc1ccc(OC)c(OC)c1)C2=O</smiles>

$\mathrm{NaBH}_{4}$

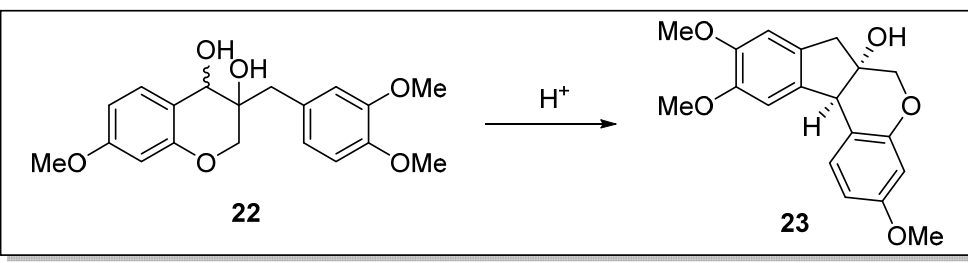

图式 4 Kirkiacharian 研究组 Brazilin 三甲醚化衍生物的合成方法

Scheme 4 Synthesis of $O$-trimethylbrazilin derivative by Kirkiacharian's group

\subsection{Kirkiacharian 研究组 Brazilin 三甲醚化衍生物的 合成方法}

该合成策略分别从中间体 17 或 19 出发, 在碱性条 件下分别与 3,4-二甲氧基苯甲醛或甲酸酯缩合得到化合 物 18 或 20, 再在钯碳/氢气的作用下还原双键得到共同 的中间体 21, 21 在羰基 $\alpha$ 位氧化形成羟基得到生源前体 苏木酮化合物 7, 化合物 7 经 $\mathrm{NaBH}_{4}$ 还原得到苏木醇化 合物 22, 而化合物 22 在酸性条件下经分子内傅克烷基 化得到消旋的 Brazilin 三甲醚化衍生物 23. 目前, 许多 关于 Brazilin 的合成都是基于对生源前体化合物 7 的合 成, 最后通过分子内傅克烷基化来构建二氢狮环. 区别 主要在于化合物 7 季碳中心的构建方法.

\subsection{Lee 研究组的合成方法}

2010 年, 美国的 Lee 研究组 ${ }^{[19]}$ 从中间体 18 出发, 首 先在双氧水作用下对双键进行环氧化, 在羰基 $\alpha$ 位引入 含氧季碳，最后通过氢化铝锂还原羰基和环氧得到苏木 醇化合物 22, 化合物 22 在高氯酸和醋酸的条件发生分 子内傅克烷基化就合成了消旋的 Brazilin 三甲醚化衍生 物 23, Brazilin 三甲醚化衍生物在盐酸吡啶条件下高温 脱甲醚就完成了 Brazilin 的消旋合成. Brazilin 再被碘氧 化就完成了 Brazilein 的首次消旋合成，合成总收率只有 $2.3 \%$ (Scheme 5). 
<smiles></smiles><smiles>COc1ccc2c(c1)OC[C@]1(O)Cc3cc(OC)c(OC)cc3[C@@H]2c2ccccc21</smiles>

Reagents and conditions: (a) $\mathrm{H}_{2} \mathrm{O}_{2}$, dioxane, r.t., 24 h, $90 \%$; (b) $\mathrm{LiAlH}_{4}$, THF, r.t., overnight, $89 \%$; (c) $\mathrm{HClO}_{4}$, acetic acid, r.t., overnight, $31 \%$; (d) pyridine- $\mathrm{HCl}, 190 \sim 200^{\circ} \mathrm{C}, 3 \mathrm{~h}, 36 \%$; (e) 1 equiv. $\mathrm{I}_{2}$ (aq.), r.t., overnight, $78 \%$.

图式 5 Lee 研究组的合成方法

Scheme 5 Synthesis by Lee's group

以上两种合成方法都是采用仿生的合成途径构建 巴西木素类天然产物四环骨架, 实现了 Brazilin 及其衍 生物的消旋合成.

\subsection{Davis 研究组的不对称合成 Brazilin 三甲醚化衍生 物的方法}

1993 年, Davis 研究组 ${ }^{[20]}$ 通过在化合物 21 的羰基 $\alpha$ 位选择性氧化形成着基，构建了手性季碳中心，而实现 了 Brazilin 三甲醚化衍生物的首次不对称合成. 该合成 方法以芳酮化合物 25 为起始原料, 经过 4 步合成中间体 21, 化合物 21 在 NaHMDS 作用下先形成烯醇盐, 然后 利用手性樟脑磺酰胺衍生物 $(+)-26$ 的氧化作用, 形成 含氧手性季碳, 以 $94 \%$ 的 $e e$ 值, $50 \%$ 的收率得到手性前 体化合物 7, 化合物 7 经 $\mathrm{NaBH}_{4}$ 还原, 分子内傅克烷基 化得到右旋的 Brazilin 三甲醚化衍生物 23 (Scheme 6).

而后续关于巴西木素类天然产物的不对称合成都
是借鉴了 Davis 课题组的合成方法. 1995 年, Merlini 研 究组 ${ }^{[21]}$ 利用此方法合成了(一)-Haematoxylin (4); 2012 年, Suzuki 研究组 ${ }^{[22]}$ 利用此方法合成了一系列 Brazilin 衍生物 30 (Scheme 7).

\subsection{Jahng 研究组不对称合成 Brazilin 的方法}

2014 年, Jahng 研究组 ${ }^{[23]}$ 报道了一种新的不对称合 成 Brazilin 的方法. 该方法同样是基于生源合成途径来 实现 Brazilin 的不对称合成，与 Davis 研究组的合成方法 不同之处在于采用 Sharpless 不对称双羟基化来构建含 氧手性季碳中心. 该合成方法不经过生源前体化合物 7, 而是通过 Sharpless 不对称双羟基化直接合成苏木醇化 合物 38, 然后发生分子内的傅克烷基化构建四环骨架, 最终完成 Brazilin 的不对称合成, 但是其 $e e$ 值较低, 仅 有 $63 \%$ (Scheme 8$)$.<smiles>COc1ccc(C(C)=O)c(O)c1</smiles><smiles>COc1ccc2c(c1)OCC(Cc1ccc(OC)c(OC)c1)C2=O</smiles>
$( \pm)-21$<smiles>COc1ccc(C[C@H]2COc3cc(C)ccc3C2=O)cc1OC</smiles>

$(-)-R-7$<smiles>COc1ccc2c(c1)OC[C@]1(O)Cc3cc(OC)c(OC)cc3[C@H]21</smiles>

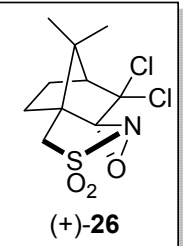

$(+)-23$ $\mathrm{OMe}$

Reagents and conditions: (a) 3,5-dimethoxy-benzaldehyde, $\mathrm{KOH}$; (b) $\mathrm{H}_{2}, \mathrm{Pd} / \mathrm{C}, 89 \%, 2$ steps; (c) $\mathrm{HCO}_{2} \mathrm{Et} / \mathrm{Na}, 89 \%$; (d) $\mathrm{Pd} / \mathrm{C}, \mathrm{H}_{2}, 98 \%$; (e) NaHMDS, (+)-26, 50\%, 94\% ee; (f) $\mathrm{NaBH}_{4}, 91 \%$; (g) $\mathrm{HCl} / \mathrm{MeOH}, 70 \%$.

图式 6 Davis 研究组的不对称合成 Brazilin 三甲醚化衍生物的方法

Scheme 6 Asymmetric synthesis of $(+)$ - $O$-trimethylbrazilin derivative by Davis's group 
<smiles>[R]c1ccc(C(C)=O)c(OC)c1[R]</smiles>

27

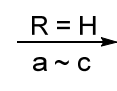

$=\mathrm{OH} \downarrow \mathrm{g} \sim \mathrm{i}$<smiles>O=C1c2ccc(OCc3ccccc3)c(OCc3ccccc3)c2OCC1Cc1ccc(OC(Br)(Br)c2ccccc2)c(OCc2ccccc2)c1</smiles>

31<smiles>O=C1c2ccccc2OCC1Cc1cccc(OCc2ccccc2)c1</smiles>

28

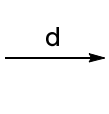<smiles>C[C](C)Cc1cccc(CC2(O)COc3ccccc3C2=O)c1</smiles><smiles>Oc1cccc2c1OC[C@@]1(O)Cc3ccccc3[C@H]21</smiles>

Deoxy-Brazilin (30)<smiles></smiles>

32<smiles>Oc1cc2c(cc1O)[C@H]1c3ccc(O)c(O)c3OC[C@]1(O)Cc1cccc(OCc3ccccc3)c1OC2</smiles>

33

(-)-Haematoxylin (4)

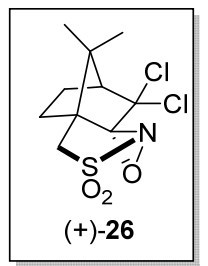

Reagents and conditions: (a) 3-benzyloxy-benzaldehyde, $\mathrm{HCl}$, EtOH, r.t., $76 \%$; (b) $\mathrm{H}_{2}$, Pd, EtOAc, r.t., $58 \%$; (c) $\mathrm{BnBr} \mathrm{K}_{2} \mathrm{CO}_{3}, \mathrm{DMF}^{\circ} 65{ }^{\circ} \mathrm{C}$, 91\%; (d) NaHMDS, (+)-26, THF, $-70{ }^{\circ} \mathrm{C}$, 98\%; (e) $\mathrm{NaBH}_{4}, 0{ }^{\circ} \mathrm{C} \sim$ r.t.; (f) $\mathrm{HCl}$, r.t., EtOH, $41 \%$ two steps; (g) 3,4 -dibenzyloxy-benzaldehyde, $\mathrm{HCl}$ gas, $65 \%$; (h) $\mathrm{H}_{2}$, $\mathrm{Pd} / \mathrm{C}$, EtOH, $83 \%$; (i) $\mathrm{BnBr}, \mathrm{K}_{2} \mathrm{CO}_{3}$, reflux, $10 \mathrm{~h}, 36 \%$; (j) $\mathrm{NaHMDS}$, (-)-26, THF, $-78{ }^{\circ} \mathrm{C}, 42 \%$; (k) $\mathrm{NaBH}$, EtOH; (l) $\mathrm{HCl}, \mathrm{EtOH}, 83 \%$ two steps; (m) $\mathrm{H}_{2}, \mathrm{Pd} / \mathrm{C}, 16 \%$.

图式 7 Merlini 研究组和 Suzuki 研究组不对称合成巴西木素类天然产物的方法

Scheme 7 Asymmetric synthesis of brazilin-type nature products by Merlini's group and Suzuki's group<smiles>COc1cc(CC2COc3cc(O)ccc3C2=O)ccc1O</smiles>

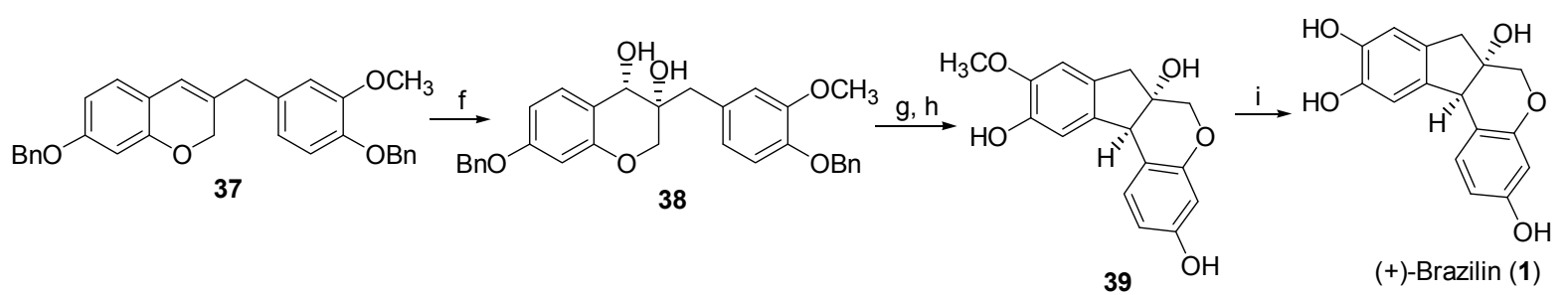

Reagents and conditions: (a) 4-hydroxy-3-methoxybenzaldehyde, $\mathrm{HCl}$ gas, $\mathrm{EtOH}, 95 \%$; (b) $\mathrm{H}_{2}, \mathrm{Pd} / \mathrm{C}$, $\mathrm{EtOH}, 98 \%$; (c) $\mathrm{BnCl}_{2} \mathrm{~K}_{2} \mathrm{CO}_{3}, 67 \%$; (d) $\mathrm{NaBH}_{4}, 88 \%$; (e) $p$-TsOH, benzene, $70 \%$; (f) AD-mix- $\alpha, 65 \%, 63 \%$ ee; (g) $\mathrm{HCl}, \mathrm{CH}_{3} \mathrm{OH}, 85 \%$; (h) $\mathrm{H}_{2}, \mathrm{Pd} / \mathrm{C}, 83 \%$; (i) $\mathrm{AlCl}_{3}, \mathrm{CH}_{2} \mathrm{Cl}_{2}, 79 \%$.

图式 8 Jahng 研究组不对称合成 brazilin 的方法

Scheme 8 Asymmetric synthesis of brazilin by Jahng's group

以上提到的关于巴西木素类天然产物的全合成研 究都是基于生源途径的一些合成策略, 都是以合成生源 前体苏木酮化合物 7 或苏木醇作为出发点, 最后通过分 子内的傅克烷基化来实现巴西木素类天然产物四环骨 架的构建, 并成功实现了 Brazilin 四环骨架的不对称构 建.

\section{2 其它方法合成巴西木素类天然产物}

\subsection{Pettus 研究组的合成方法}

Brazilin 真正严格意义上的全合成是由美国的 Pettus 研究组在 2005 年完成的(Scheme 9) ${ }^{[24]}$. 

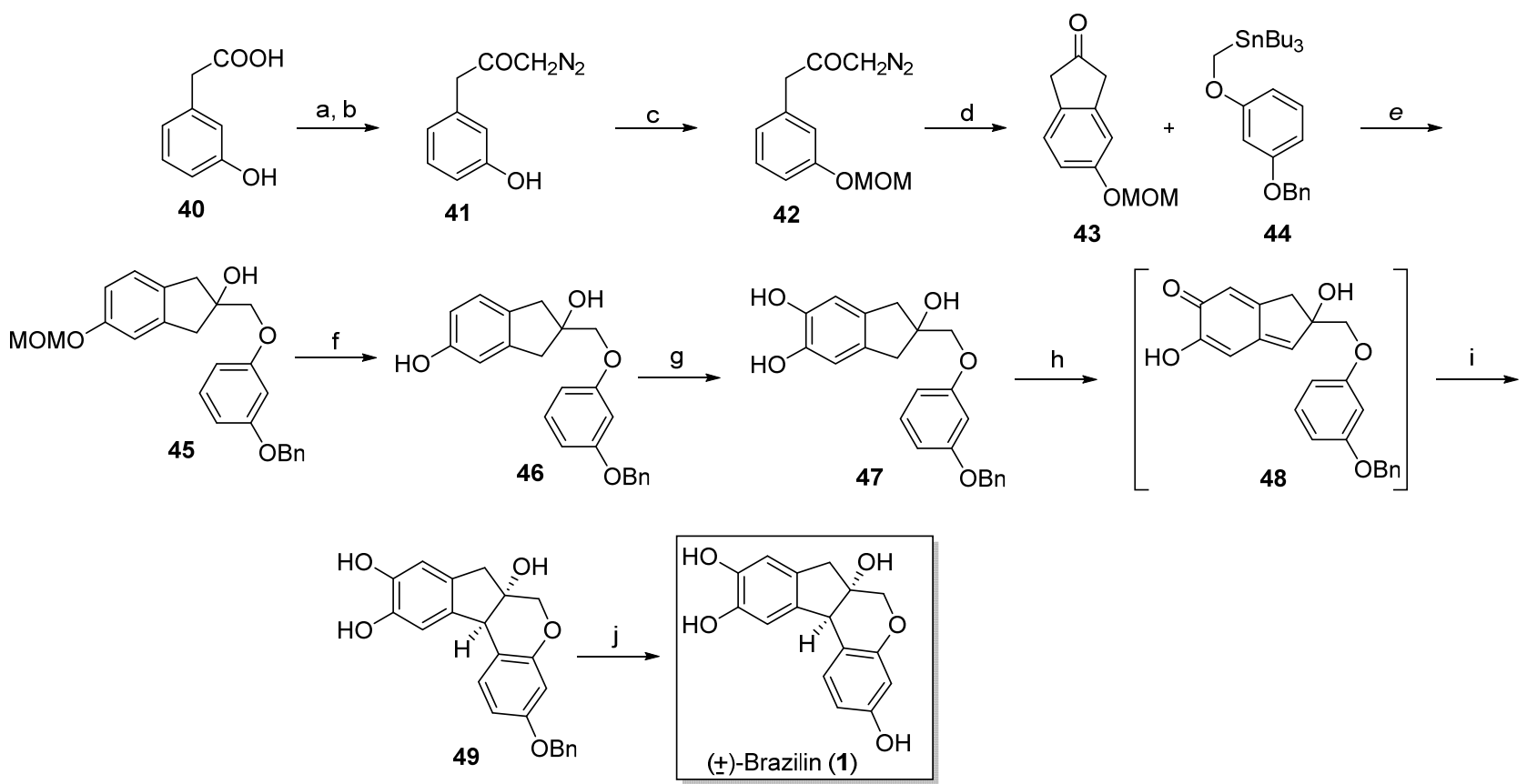

Regents and conditions: (a) (COCl) 2 , DMF, $\mathrm{CH}_{2} \mathrm{Cl}_{2}$; (b) $\mathrm{CH}_{2} \mathrm{~N}_{2}, 68 \%$ two steps; (c) $\mathrm{K}_{2} \mathrm{CO}_{3}, \mathrm{MOMCl}, n-\mathrm{Bu}_{4} \mathrm{NI}, 76 \%$; (d) $\mathrm{Rh}(\mathrm{OAc})_{2} \cdot \mathrm{H}_{2} \mathrm{O}, \mathrm{CH}_{2} \mathrm{Cl}_{2}$, 74\%; (e) n-BuLi, HF, $-100{ }^{\circ} \mathrm{C}, 61 \%$; (f) HCl, MeOH, 95\%; (g) IBX, DMF, 2 h, 58\%; (h) PIFA, 95\%; (i) Lil, 70\%; (j) Pd/C, $\mathrm{H}_{2}$, quantitative.

图式 9 Pettus 研究组的合成方法

Scheme 9 Synthesis by Pettus's group

该合成方法以间羟基苯乙酸 40 为起始原料, 首先 制备重氮酮化合物 41, 对 41 酚羟基进行 MOM 保护后, 再在金属铑催化下进行碳氢插入得到 2-狮酮化合物 43. 有机锡试剂 44 和金属锂试剂首先在低温条件下进行锂 交换, 再对化合物 43 进行亲核加成得到化合物 $\mathbf{4 5}$, 化 合物 45 经脱 MOM 保护, IBX 氧化得到邻二酚化合物 $\mathbf{4 7}$, 47 可被 PIFA 氧化为醌, 醌可发生互变异构得异构体 $\mathbf{4 8}$, 48 在碘化锂作用下发生分子内傅克烷基化生成化合物 49, 从而成功构建了 Brazilin 的四环骨架. 化合物 49 脱 芐基保护就完成了 Brazilin (1)的消旋合成, Pettus 的合 成总共经过 9 步, 以 $8.5 \%$ 的总收率获得 Brazilin.

\subsection{Rai-Shung Liu 研究组的合成方法}

2008 年, 台湾清华大学的 Liu 研究组 ${ }^{[25]}$ 发展了一种 利用金催化的脱氧 Nazarov 环化, 选择性地合成多取代 环戊烯的方法, 并考察了这一方法在巴西木素类天然产 物四环骨架构建中的应用. 该方法利用化合物 $\mathbf{5 0}$ 在三 苯基膦氯化金的催化作用下发生脱氧 Nazarov 环化成功 构建了巴西木素类天然产物的四环骨架, 进一步脱甲醚 和硅醚保护基就完成 $( \pm)$-Brazilane (6)的全合成. 同样 的, 以化合物 62 为原料, 就可顺利合成 Haematoxylane 四甲基衍生物 53 (Scheme 10). 这一合成方法高效、简 洁, 一步同时构建了巴西木素类天然产物的两个环系, 为构建巴西木素类天然产物四环骨架提供了一种高效
的合成方法.

\section{3 张洪涁研究组关于巴西木素类天然产物及其类似 物的合成方法}

2011 年, 我们研究组 ${ }^{[26]}$ 报道了一种普适性的构建 巴西木素类似物四环骨架的合成方法, 合成了一系列巴 西木素衍生物, 并对其生物活性进行了篮选, 发现部分 化合物对结肠癌(HT29)、肺癌(A549)、白血病(HL60)和 白血病(K562)细胞株具有很好的抑制活性. 合成策略主 要通过分子内傅克烷基化构建含杂原子的六元环, 合成 方法从 3,4-二甲氧基苯丙酸(54)出发, 经过分子内傅克 酰基化合成茚酮化合物 55, 55 进一步反应制备 $\alpha, \beta$-不饱 和酸 56, 化合物 56 和苯酚或苯胺缩合就得到酯或酰胺 化合物 57, 化合物 57 经双键双羟基化、分子内傅克烷 基化就合成了巴西木素类似物 59 (Scheme 11).

而从化合物 60 出发, 先发生胺解得到化合物 61 , 化合物 61 在七水三氯化铈、氧气的作用下在双羰基 $\alpha$ 位氧化产生羟基，再对酮羰基进行还原得到二醇化合物 63, 再在路易斯酸催化下发生分子内傅克烷基化就合成 了巴西木素含氮衍生物 64. 而化合物 61 不经氧化, 直 接还原羰基为醇, 还原酰胺为胺, 再经路斯酸催化发生 分子内傅克烷基化就合成了巴西木烷含氮衍生物 62 (Scheme 12). 


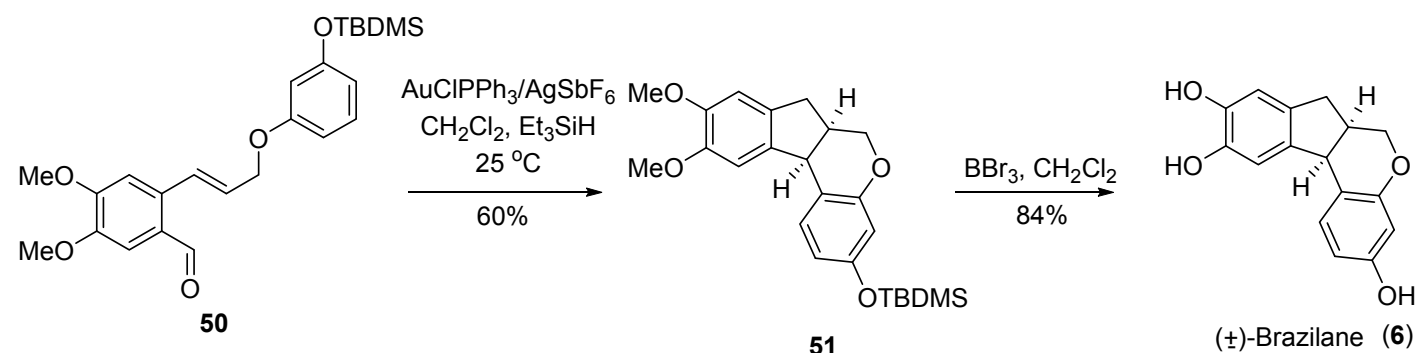<smiles>COc1cc(C=O)c(/C=C/COc2cccc(OC)c2OC)cc1OC</smiles>

图式 10 Rai-Shung Liu 研究组的合成方法

Scheme 10 Synthesis by Rai-Shung Liu's group<smiles>COc1ccc(CCC(=O)O)cc1OC</smiles>

54<smiles>COc1cc2c(cc1OC)C([In]C)CC2=O</smiles>

55<smiles>COc1cc2c(cc1OC)CC(C(=O)O)=C2</smiles>

56<smiles>[X]C(=O)C1=Cc2cc(OC)c(OC)cc2C1</smiles>

57 $\mathrm{X}=\mathrm{N}, \mathrm{O}$

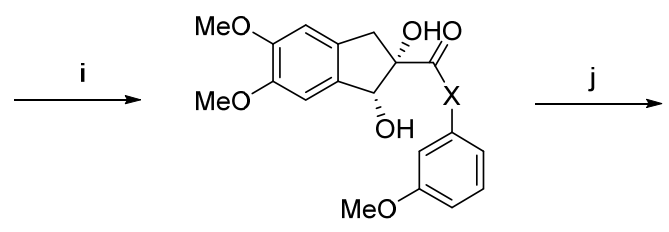

58

$\mathrm{X}=\mathrm{N}, \mathrm{O}$

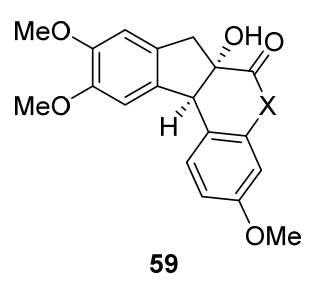

$\mathrm{X}=\mathrm{N}, \mathrm{O}$

Reagents and conditions: (a) $(\mathrm{COCl})_{2}, \mathrm{DMF}, \mathrm{CH}_{2} \mathrm{Cl}_{2}$; (b) $\mathrm{AlCl}_{3}, \mathrm{CH}_{2} \mathrm{Cl}_{2}, 68 \%$ two steps; (c) $\mathrm{NaH}, \mathrm{CO}(\mathrm{OMe})_{2}, 92 \%$; (d) $\mathrm{NaBH}$, $\mathrm{MeOH}$; (e) PPTS, toluene, $70 \%$ two steps; (f) $\mathrm{KOH}, \mathrm{MeOH}, \mathrm{H}_{2} \mathrm{O}, 66 \%$; (g) $\left(\mathrm{COCl}_{2}\right.$, DMF, $\mathrm{CH}_{2} \mathrm{Cl}_{2}$; (h) $m$-methoxyphenol or $m$-methoxyaniline, Et $\mathrm{N}_{3} \mathrm{~N}$, $\mathrm{CH}_{2} \mathrm{Cl}_{2}$; (i) $\mathrm{OsO}_{4}, \mathrm{NMO}$, acetone $/ \mathrm{H}_{2} \mathrm{O}(\mathrm{V}: \mathrm{V}=9: 1)$; (j) $\mathrm{BF}_{3} \cdot \mathrm{OEt}_{2},-78{ }^{\circ} \mathrm{C}$.

图式 11 Brazilin 类似物 59 的合成

Scheme 11 Synthesis of brazilin-like compounds 59

2013 年, 我们研究组在此基础上进一步发展了一 种高效、普适的不对称合成巴西木素类天然产物的合成 方法, 并完成了 $(+)$-Brazilin、( - )-Brazilein 和 $(+)$-Brazilide A 三个天然产物的首次不对称全合成 ${ }^{[27]}$.

合成方法仍然以 3,4-二甲氧基苯丙酸(54)为起始原 料合成化合物 65, 然后利用二异丁基氢化铝(DIBAL-H) 对酯基进行还原得荫甲醇化合物 66, 以四步 $61 \%$ 的总 收率获得化合物 66. 化合物 66 与化合物 67 经 Mistnobu 偶联得化合物 68 , 化合物 68 在化合物 67 存在的条件下 经 Sharpless 不对称双羟基化成功构建含氧手性季碳,
从而以 $85 \%$ 的收率, $81 \%$ 的 $e e$ 值获得化合物 69. 化合物 69 在 PPTS 的作用下发生分子内的傅克烷基化成功构建 巴西木素类天然产物的四环骨架. 化合物 70 水解脱苯 甲酰基保护得到关键中间体 71, 经两次重结晶后, 其 $e e$ 值可达到 $99.5 \%$. 化合物 71 在三溴化硼作用脱甲醚保护 生成 Brazilin (1), 共 9 步, 14\%的总收率. Brazilin (1)经 IBD 氧化生成 Brazilein (2) (Scheme 13). 这是首次对 Brazilin 和 Brazilein 不对称全合成的报道, 合成方法高 效、简洁, 并具有很好的普适性. 

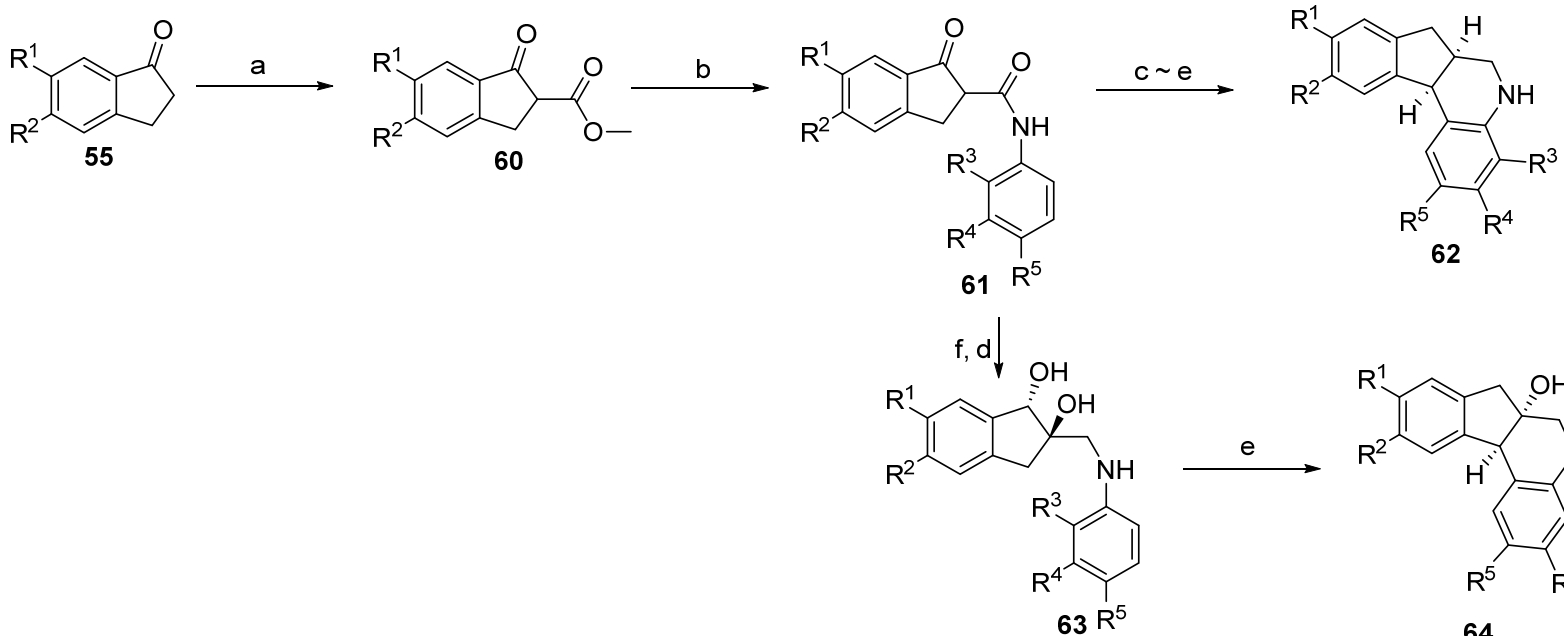
$\mathrm{CeCl}_{3} \cdot 7 \mathrm{H}_{2} \mathrm{O}, \mathrm{O}_{2}$

图式 12 Brazilin 类似物 62 和 64 的合成

Scheme 12 Synthesis of brazilin-like compounds 62 and 64
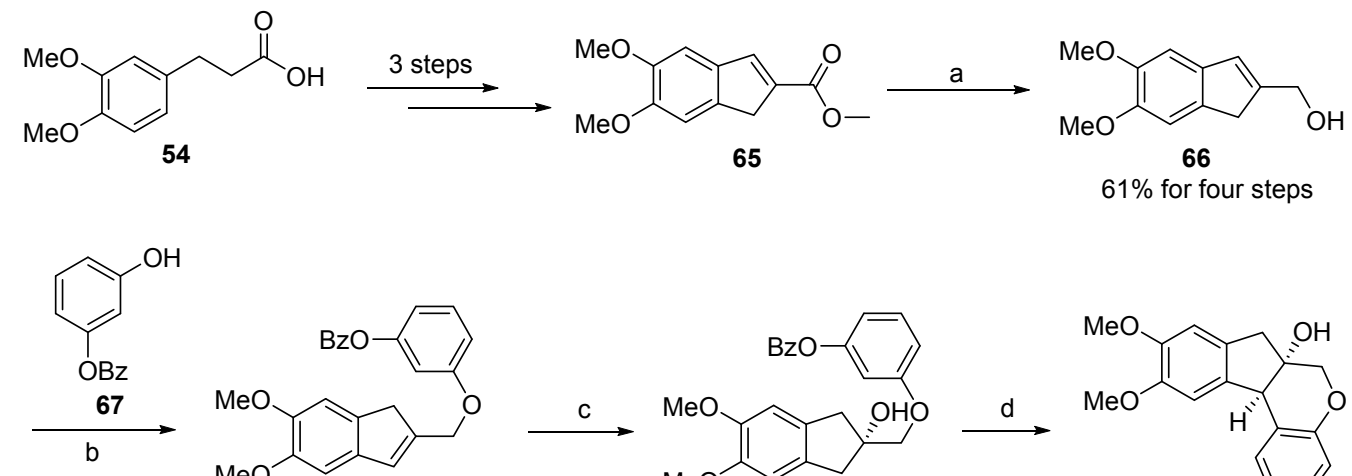<smiles>COc1cc2cc(c1)CC(COc1cccc(OC(=O)c3ccccc3)c1)C2</smiles>

68

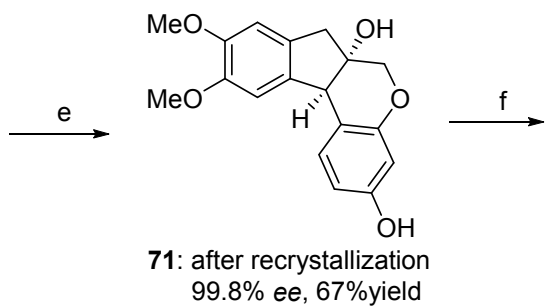

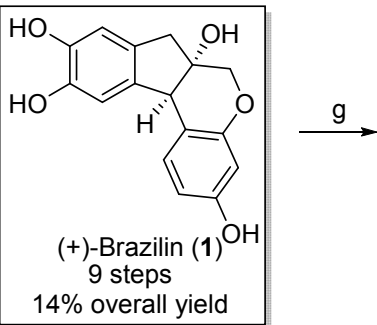

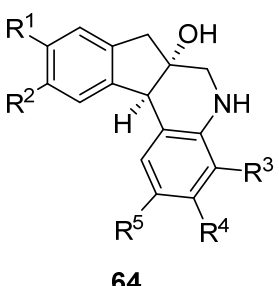

64

(d) $\mathrm{LiAlH}_{4}, \mathrm{THF} ;$ (e) $\mathrm{BF}_{3} \cdot \mathrm{OEt}_{2}, \mathrm{CH}_{2} \mathrm{Cl}_{2}$; (f)

Reagents and conditions: (a) DIBAL- $\mathrm{H}, \mathrm{CH}_{2} \mathrm{Cl}_{2},-78{ }^{\circ} \mathrm{C}, 91 \%$; (b) DEAD, $\mathrm{Ph}_{3} \mathrm{P}, \mathrm{THF},-78{ }^{\circ} \mathrm{C}, 60 \%$; (c) AD-mix- $\beta, \mathrm{OsO}_{4}, \mathrm{MeSO}_{2} \mathrm{NH}_{2}$, $t-\mathrm{BuOH} / \mathrm{H}_{2} \mathrm{O} / \mathrm{CH}_{2} \mathrm{Cl}_{2}, 67,3 \mathrm{~d}$, 85\%, 81\% ee; (d) PPTS, toluene, $90{ }^{\circ} \mathrm{C}, 80 \%$; (e) $\mathrm{LiOH}, \mathrm{THF} / \mathrm{MeOH}, 92 \%$; (f) $\mathrm{BBr}_{3}, \mathrm{CH}_{2} \mathrm{Cl}_{2}, 81 \%$; (g) Phl(OAc) THF, $0{ }^{\circ} \mathrm{C}, 76 \%$.

图式 13 (+)-Brazilin 和( - -)-brazilein 的全合成

Scheme 13 Total synthesis of $(+)$-brazilin and $(-)$-brazilein

在完成 Brazilin 全合成的基础上, 本研究组从关键 中间体 Brazilin二甲醚化衍生物 71 出发, 利用芳环上电 子云密度的差异性, 在液氨、金属锂的作用下, 选择性 地对二甲氧基取代的芳环进行 Birch 还原得到化合物
72, 对 72 的酚差基进行苯甲酰基保护得到化合物 73 . 化合物 73 在 PPTS 存在的条件下, 利用 $\mathrm{O}_{3}$ 对富电子、 位阻小的双键进行选择性地切断可以高收率地得到关 键中间体 74. 关键中间体 74 利用羟基的导向作用, 对 
双键进行环氧化可以单一地得到环氧化合物 75, 化合 物 75 在路易斯酸三氟化硼乙醚的作用下形成关键骨 架一一双内酯环, 令人遗憾的是, 其双内酯环的构型刚 好与天然产物构型相反, 最终脱除保护得到的是 Brazilide A 的类似物 77 (Scheme 14).

分析可能的原因, 我们推测是三氟化嗍乙醚和环氧 络合后, 甲酯只能从环氧位阻较小的一侧进攻. 当环氧 和差基同侧时, 得到的就是构型相反的化合物, 因此调 整环氧的构型就有可能实现 Brazilide A 的全合成 (Scheme 15).

为了实现对环氧构型的调控, 首先对化合物 74 的
叔羟基进行 TBS 保护, 然后进行环氧化就可得到一对 非对映异构体 82 和 83 , 化合物 82 在路易斯酸三氟化硼 乙醚的作用下形成双内酯环，脱保护就可以得到 77. 化 合物 83 在路易斯酸三氟化硼乙醚作用下, 生成双内酯 结构, 在硫酸作用下一步脱除 TBS 保护和 $\mathrm{Bz}$ 保护, 生 成 Brazilide A. 该合成方法首次完成了 Brazilide A 的不 对称全合成, 共经历了 16 步反应, 总收率 $1.3 \%$ (Scheme 16). 通过单晶衍射实验确认了 Brazilide A 的绝对构型. 有趣的是，只要控制好环氧的构型就可以实现对双内酯 环构型的绝对控制，这对进一步考察 Brazilide A 的生 源合成途径具有重要的意义.

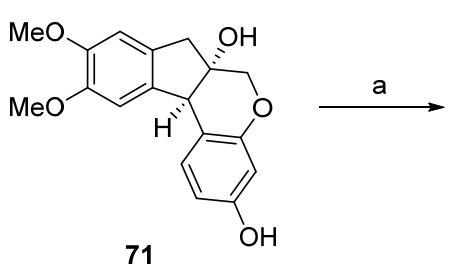<smiles>COC1=C(OC)CC2=C(C1)C[C@]1(O)COc3cc(O)ccc3[C@H]21</smiles><smiles>COC1=C(OC)CC2=C(C1)[C@@H]1C[C@@]2(O)COc2cc(OC(C)(C)C)ccc21</smiles><smiles>[R6]Oc1ccc2c(c1)OC[C@]1(O)CC(CC(=O)OC)=C(CC(=O)OC)[C@H]21</smiles>
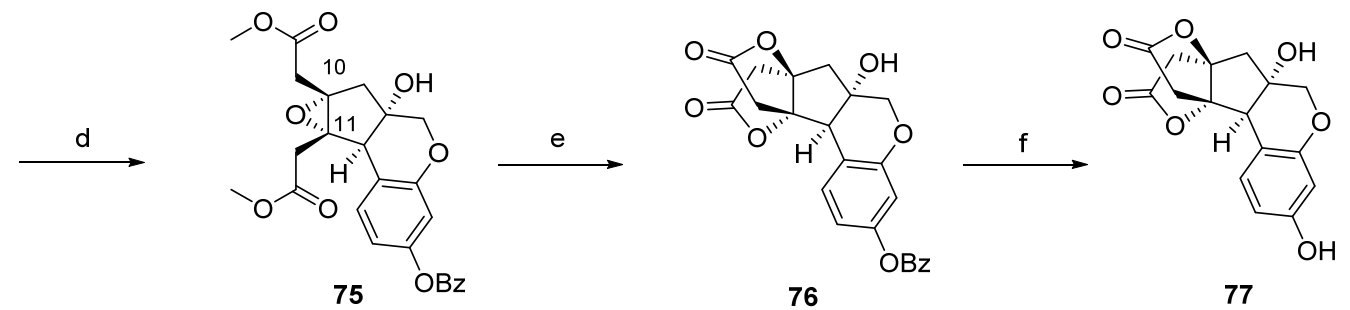

77

Reagents and conditions: (a) $\mathrm{Li}, \mathrm{NH}_{3}(\mathrm{I}), \mathrm{EtOH}, \mathrm{THF},-78{ }^{\circ} \mathrm{C}$; (b) $\mathrm{BzCl}, \mathrm{Et}_{3} \mathrm{~N}$, DMAP, DCM; (c) $\mathrm{O}_{3}, \mathrm{PPTS}, \mathrm{EtOAc/MeOH}, 50 \% 3 \mathrm{steps}$; (d) $m$-CPBA, $\mathrm{CH}_{2} \mathrm{Cl}_{2}, 92 \%$; (e) $\mathrm{BF}_{3} \cdot \mathrm{OEt}_{2}, \mathrm{CH}_{2} \mathrm{Cl}_{2}, 84 \%$; (f) $65 \% \mathrm{H}_{2} \mathrm{SO}_{4}, \mathrm{AcOH}, 110{ }^{\circ} \mathrm{C}, 90 \%$.

图式 14 Brazilide A 类似物的合成

Scheme 14 Synthesis of brazilide A-like compound
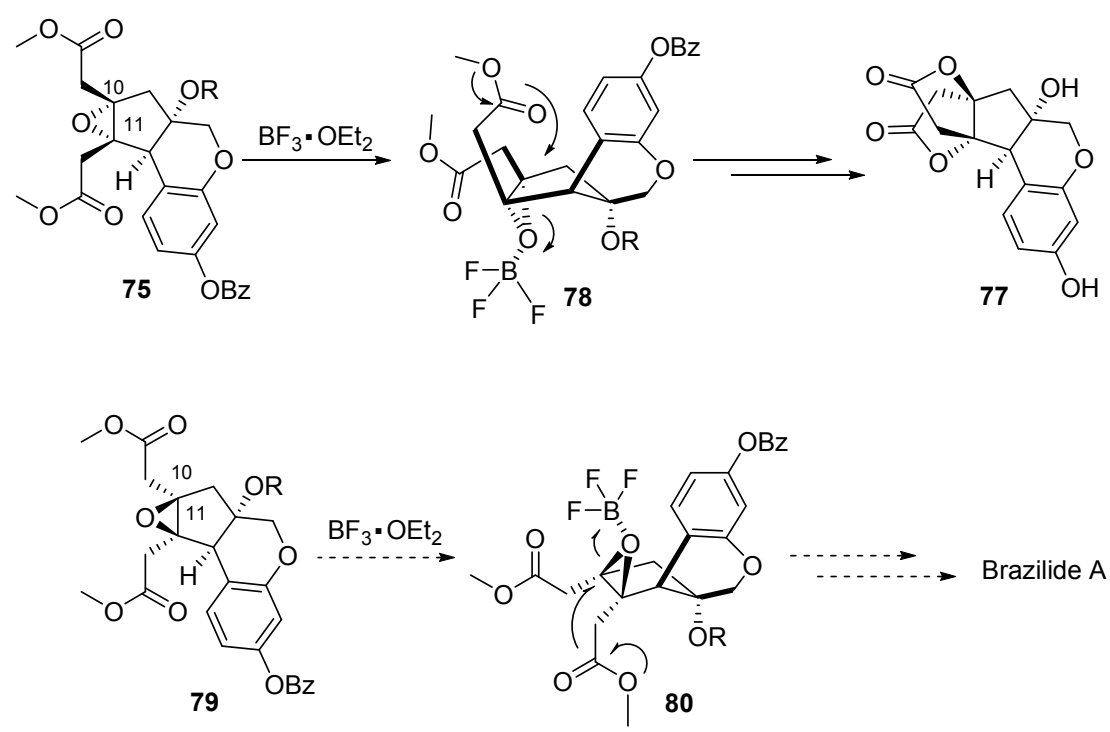

图式 15 双内酯化的可能途径

Scheme 15 Possible pathway for bis-lactonization 

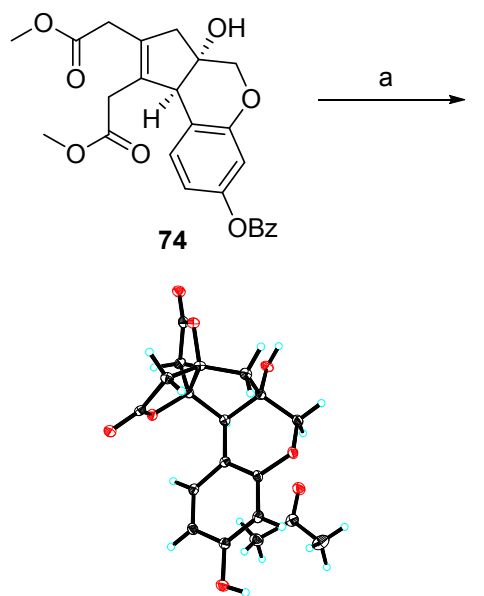

(+)-Brazilide A (3)
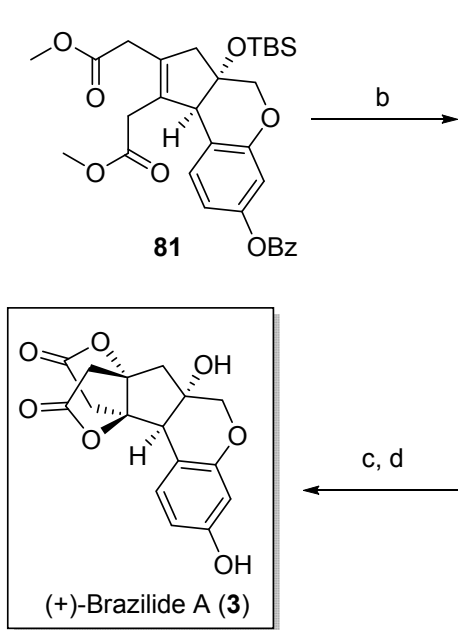

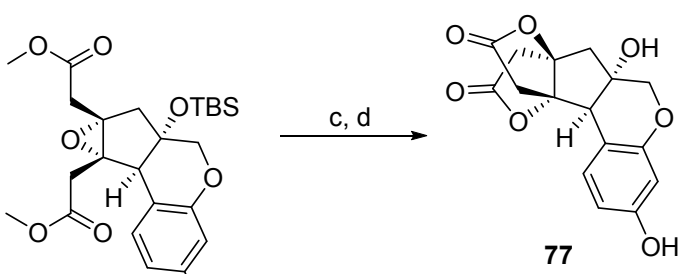

$8263 \% \quad \mathrm{OBz}$

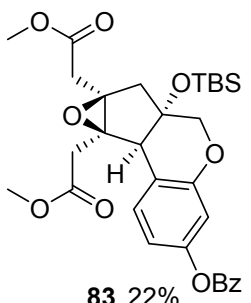

Reagents and conditions: (a) TBSOTf, 2,6-lutidine, $\mathrm{CH}_{2} \mathrm{Cl}_{2}, 85 \%$; (b) $m$-CPBA, $\mathrm{CH}_{2} \mathrm{Cl}_{2}, 32{ }^{\circ} \mathrm{C}, 3 \mathrm{~d}, 8263 \%, 8322 \%$; (c) $\mathrm{BF}_{3} \cdot \mathrm{OEt}_{2}, \mathrm{CH}_{2} \mathrm{Cl}_{2}$; (d) $65 \% \mathrm{H}_{2} \mathrm{SO}_{4}, \mathrm{AcOH}, 110{ }^{\circ} \mathrm{C}, 7780 \% 2$ steps, $382 \% 2$ steps.

图式 $16(+)$-Brazilide A 的全合成

Scheme 16 Total synthesis of $(+)$-brazilide A

到目前为止，该合成路线是唯一完成 Brazilin、 Brazilein、Brazilide A 不对称全合成的方法, 合成路线高 效、简洁, 具有很好的普适性, 可进一步用于巴西木素 类天然产物及其类似物的合成, 从而为药物化学研究提 供物质基础.

\section{4 秦红波研究组的合成方法}

2013 年, 秦红波研究组 ${ }^{[28]}$ 也报道了与本研究组类 似的构建 Brazilin 四环骨架的方法, 更高效地完成了 Brazilin 的消旋合成, 仅以 8 步反应, $23 \%$ 的总收率获得 Brazilin. 该合成方法也以䒢酮化合物 55 为起始原料, 经过三步合成狮甲醇 66, 化合物 66 在进行间氯过氧苯 甲酸 $(m-\mathrm{CPBA})$ 环氧化时, 发生环氧开环得到邻二醇 85 . 化合物 85 中的伯羟基经磺酸酯活化后, 在碳酸铯碱性 作用下与间苯二酚缩合形成醚 $\mathbf{8 7}$, 化合物 87 在三氟化 嗍乙醚作用下发生分子内傅克烷基化就构建了 Brazilin 的四环骨架 71, 化合物 71 经三溴化硼脱甲醚保护就完 成了 Brazilin 的消旋合成(Scheme 17).

\subsection{Yadav 研究组的合成方法}

2014 年, Yadav 研究组 ${ }^{[29]}$ 也利用两次傅克烷基化作 为关键步骤成功构建了 Brazilin 的四环骨架, 并完成了 Brazilin 和 Brazilane 的消旋合成. 该合成方法通过化合 物 89 在醋酸钯的催化下发生分子内傅克烷基化, 高效 地构建䒢环, 得到化合物 $\mathbf{9 0}$, 化合物 90 经还原、保护、 双着基化得到傅克反应前体 $\mathbf{9 2}$, 化合物 92 与间二苄氧 基苯在三氟化嗍乙醚作用下发生分子间的傅克烷基化 得到关键中间体 93 , 化合物 93 经简单的几步转化, 最
后形成醚键构建 Brazilin 的四环骨架 $\mathbf{9 5}$, 化合物 $\mathbf{9 5}$ 经脱 保护、还原就可以完成 Brazilin 和 Brazilane 的全合成 (Scheme 18).

\section{$2.6 \mathrm{Kim}$ 研究组的合成方法}

2015 年, Kim 研究组 ${ }^{[30]}$ 发展了一种高效地构建 Brazilin 核心骨架的方法，以三氟甲磺酸铟催化的炔-醛 复分解反应成功构建苯并吡喃环结构, 进一步通过双羟 基化、脱氧和分子内傅克烷基化构建 Brazilin 四环骨架， 最终以 9 步反应, $70 \%$ 的总收率完成了 Brazilin 的消旋合 成(Scheme 19).

该合成方法首先利用 Mistnobu 反应将化合物 97 和 98 偶联制备得到中间体 99, 99 在三氟甲磺酸铟的催化 作用下发生炔一醛复分解反应，构建关键的苯并吡喃环 系，得到化合物 100. 化合物 100 通过双键双差基化构 建含氧季碳中心，然后对邻二醇进行保护，再利用硼氢 化钠还原宸基成羟基，最后利用 Barton-McCombie 去氧 反应脱除羟基得到化合物 103. 化合物 103 经脱保护、 分子内傅克烷基构建 Brazilin 四环骨架，最后脱甲基完 成 Brazilin 的消旋合成. 该研究组也对其不对称合成进 行了研究, 在构建含氧季碳时, 利用 Sharpless 不对称双 羟基化来引入手性季碳中心，从而可以进一步完成 Brazilin 的不对称合成.

Kim 研究组的合成方法是目前报道的最高效的合 成方法，其步骤简洁，合成效率高，可以以 $70 \%$ 的总收 率获得 Brazilin 的消旋体, 这为 Brazilin 的进一步开发和 利用提供了非常好的合成途径. 


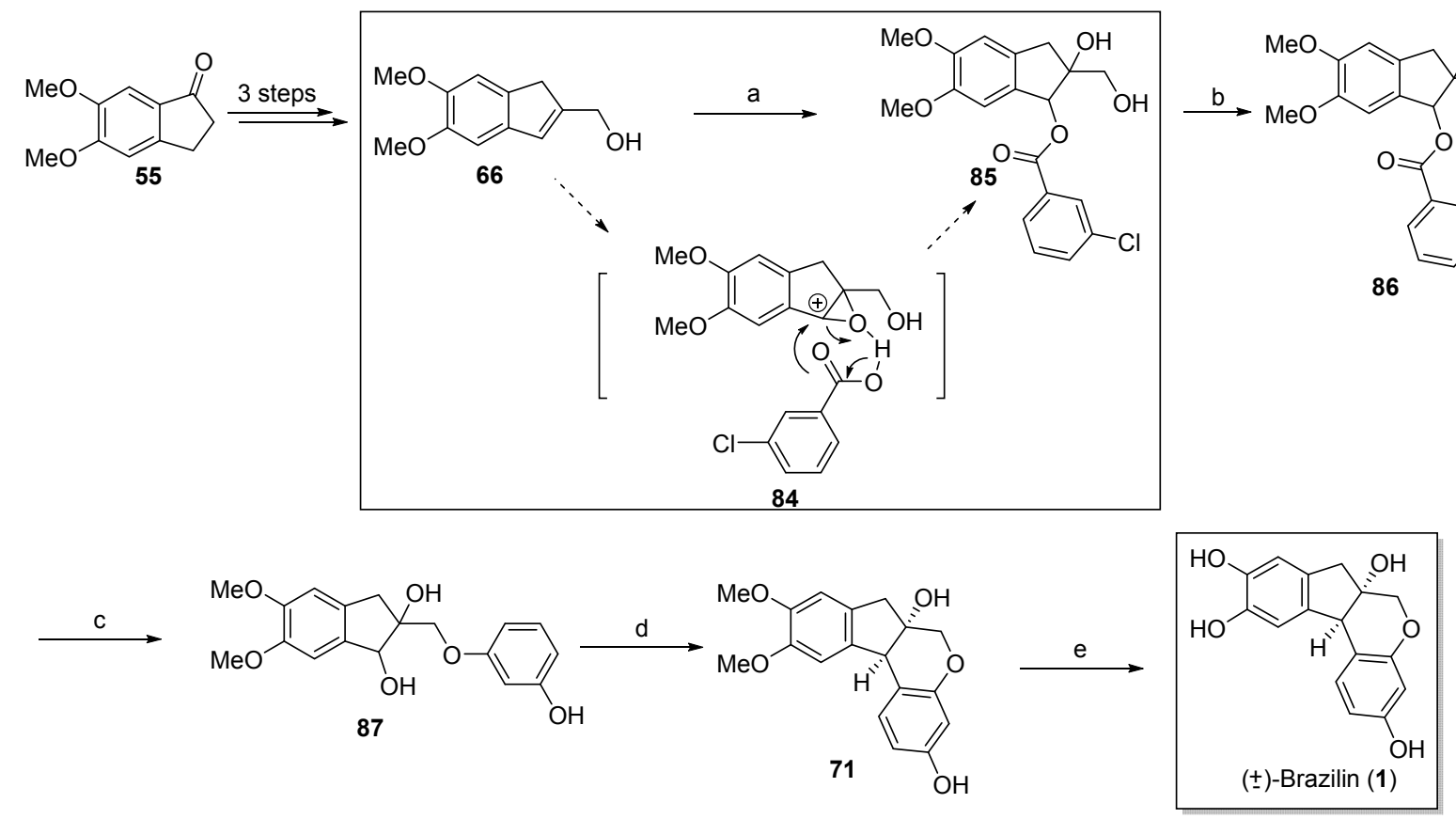

Reagents and conditions: (a) $m$-CPBA, $\mathrm{CH}_{2} \mathrm{Cl}_{2}, 0{ }^{\circ} \mathrm{C}$, r.t., $69 \%$; (b) $\mathrm{TsCl}, \mathrm{Et}_{3} \mathrm{~N}, \mathrm{Me}_{3} \mathrm{~N} \cdot \mathrm{HCl}$, toluene, $80{ }^{\circ} \mathrm{C}, 75 \%$; (c) Reaorcine, $\mathrm{Cs}_{2} \mathrm{CO}_{3}, \mathrm{DMF}$, $60 \sim 85{ }^{\circ} \mathrm{C}, 94 \%$; (d) $\mathrm{BF}_{3} \cdot \mathrm{OEt}_{2}, \mathrm{CH}_{2} \mathrm{Cl}_{2},-78 \sim 0{ }^{\circ} \mathrm{C}, 76 \%$; (e) $2 \mathrm{~mol} / \mathrm{L} \mathrm{BBr}_{3} / \mathrm{CH}_{2} \mathrm{Cl}_{2}, \mathrm{CH}_{2} \mathrm{Cl}_{2},-78{ }^{\circ} \mathrm{C} \sim$ r.t., $88 \%$.

图式 17 秦红波研究组的合成方法

Scheme 17 Synthesis by Hong-Bo Qin's group<smiles>C=C(OCC)C(OCC)C(C(=O)OCC)c1ccc(OC)c(OC)c1</smiles>

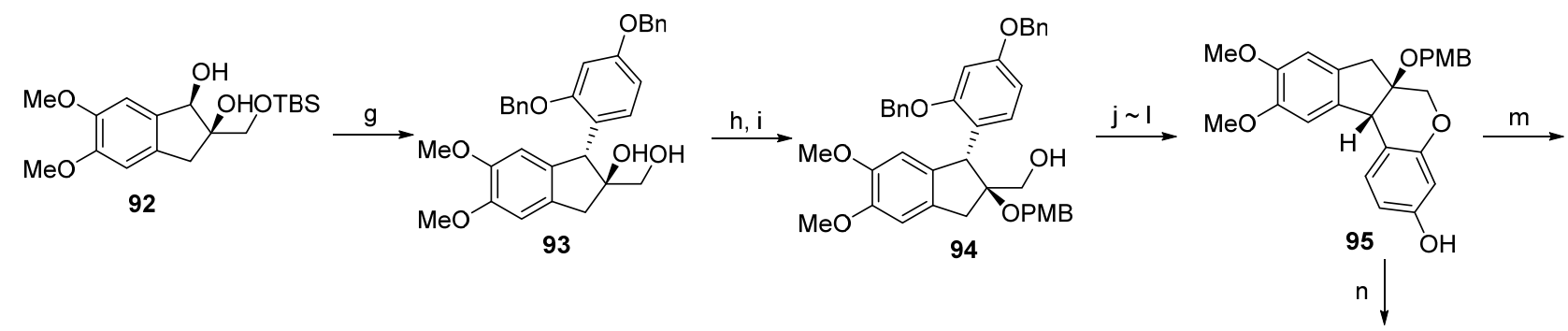<smiles>COc1cc2c(cc1OC)[C@H]1c3ccc(O)cc3OC[C@]1(O)CO2</smiles>

( \pm )-Braziline (1)<smiles>Oc1ccc2c(c1)OC[C@H]1Cc3cc(O)c(O)cc3[C@H]21</smiles>

(士)-Brazilane (6)<smiles>COc1cc2c(cc1OC)[C@H]1COc3cc(O)ccc3[C@H]1CO2</smiles>

96

Reagents and conditions: (a) DABCO, EtOAc, sonication, $100 \mathrm{~W}, 63 \%$; (b) $\mathrm{Ac}_{2} \mathrm{O}$, pyridine, DMAP, $\mathrm{CH}_{2} \mathrm{Cl}_{2}, 0{ }^{\circ} \mathrm{C} \sim$ r.t., $10 \mathrm{~h}, 90 \%$; (c) $\mathrm{Pd}(\mathrm{OAc})_{2}$, $\mathrm{PPh}_{3}, \mathrm{~K}_{2} \mathrm{CO}_{3}$, dioxane, $110{ }^{\circ} \mathrm{C}, 10 \mathrm{~h}, 67 \%$; (d) DIBAL-H, $\mathrm{CH}_{2} \mathrm{Cl}_{2},-15{ }^{\circ} \mathrm{C}, 1 \mathrm{~h}, 92 \%$; (e) TBSCl, Imidazole, $0{ }^{\circ} \mathrm{C}, 0.5 \mathrm{~h}, 92 \%$; (f) OsO ${ }_{4}, \mathrm{NMO}$, acetone $/ \mathrm{H}_{2} \mathrm{O}(\mathrm{V}: \mathrm{V}=9: 1)$, r.t., $10 \mathrm{~h}, 90 \%$; (g) 1,3-bis(benzyloxy)benzene, $\mathrm{BF}_{3} \cdot \mathrm{OEt}_{2}, \mathrm{CH}_{2} \mathrm{Cl}_{2},-78{ }^{\circ} \mathrm{C}, 30$ min, $90 \%$; (h) anisaldehyde dimethyl acetal, CSA, $\mathrm{CH}_{2} \mathrm{Cl}_{2}, 0{ }^{\circ} \mathrm{C} \sim$ r.t., $1 \mathrm{~h}, 94 \%$; (i) DIBAL-H, $\mathrm{CH}_{2} \mathrm{Cl}_{2},-20{ }^{\circ} \mathrm{C}, 1 \mathrm{~h}, 95 \%$; (j) $\mathrm{MsCl}, \mathrm{Et}_{3} \mathrm{~N}, 0{ }^{\circ} \mathrm{C}, 30 \mathrm{~min}, 97 \%$; (k) Raney Ni, $\mathrm{H}_{2}$, $\mathrm{EtOH}$, r.t., $1 \mathrm{~h}$; (I) $\mathrm{NaH}$, THF, $0{ }^{\circ} \mathrm{C}, 30 \mathrm{~min}, 90 \%$ two steps; (m) $\mathrm{HCl}, \mathrm{CH}_{2} \mathrm{Cl}_{2}, 0{ }^{\circ} \mathrm{C}, 1 \mathrm{~h}, 60 \%$; (n) $\mathrm{Pd}(\mathrm{OH})_{2} / \mathrm{C}, \mathrm{H}_{2}$, EtOAc, $2 \sim 4$ h, r.t., quant.; (o) $\mathrm{BBr}_{3}, \mathrm{CH}_{2} \mathrm{Cl}_{2},-78{ }^{\circ} \mathrm{C} \sim$ r.t., 2 h, $92 \%$.

图式 18 Yadav 研究组的合成方法

Scheme 18 Synthesis by Yadv's group 
<smiles>COc1ccc(C(=O)c2ccc(OC)c(OC)c2)c(OC)c1</smiles><smiles>COc1ccc2c(c1)OCC(C(=O)c1ccc(OC)c(OC)c1)[C@H]2O</smiles><smiles>COc1ccc2c(c1)OCC(C(=O)c1ccc(OC)c(OC)c1)C2OC(C)(C)C</smiles><smiles>COc1ccc2c(c1)OC[C@H](Cc1ccc(OC)c(OC)c1)[C@H]2OC(C)(C)C</smiles><smiles>Oc1ccc2c(c1)OC[C@]1(O)Cc3cc(O)c(O)cc3[C@H]21</smiles>

$( \pm)$-Brazilin (1)

Reagents and conditions: (a) $\mathrm{PPh}_{3}$, DIAD, $\mathrm{CH}_{2} \mathrm{Cl}_{2}, 100 \%$; (b) $\ln (\mathrm{OTf})_{3},\left(\mathrm{CH}_{2} \mathrm{Cl}\right)_{2}, 100 \%$; (c) $\mathrm{OsO}_{4}, \mathrm{NMO}$, acetone/ $\mathrm{H}_{2} \mathrm{O}, 89 \%$; (d) 2,2-Dimethoxypropane, acetone, PTSA, 100\%; (e) $\mathrm{NaBH}_{4}, 100 \%$; (f) $\mathrm{NaH}, \mathrm{CS}_{2}, \mathrm{Mel}, 100 \%$; (g) $\mathrm{Bu}_{3} \mathrm{SnH}, \mathrm{AlBN}, 100 \%$; (h) $17 \% \mathrm{HCl} / \mathrm{MeOH}, 90{ }^{\circ} \mathrm{C}$, $95 \%$; (i) $\mathrm{BBr}_{3}, 83 \%$.

图式 19 Kim 研究组的合成方法

Scheme 19 Synthesis by Kim's group

\section{3 结语与展望}

天然产物的全合成是有机合成的重要内容, 是有机 合成化学发展水平的集中体现, 探索高效、简洁和绿色 经济的合成方法为天然产物的开发和利用提供了重要 保证. 尽管巴西木素类天然产物的合成自 20 世纪 70 年 代起，已开展了多年的研究，也有许多关于其合成的报 道，但是高效、简洁和普适性好的不对称合成方法仍值 得期待. 巴西木素类天然产物具有广泛的生物活性和开 发前景, 开展关于巴西木素类天然产物及其衍生物的合 成和构效关系的相关研究仍然具有非常重要的意义.

\section{References}

[1] Robinson, R. Bull. Soc. Chim. Fr. 1958, 125.

[2] Masahiro, N.; Seiji, N. Chem. Pharm. Bull. 1990, 38, 1490.

[3] Michio, N.; Hiroyuki, N.; Mariko, N. Chem. Pharm. Bull. 1987, 35, 3568 .

[4] Yang, B. O.; Ke, C. Q.; He, Z. S.; Yang, Y. P.; Ye, Y. Tetrahedron Lett. 2002, 43, 1731

[5] Kim, D. S.; Baek, N. I.; Oh, S. R.; Jung, K. Y.; Lee, I. S.; Lee, H. K. Phytochemistry 1997, 46, 177.

[6] (a) Puchtler, H.; Sweat, F. Histochemistry 1964, 4, 197.

(b) Puchtler, H.; Meloan, S. N.; Waldrop, F. S. Histochemistry 1986, 85,353

[7] Kim, B. H.; Kim, S. J.; Jeong, E. J.; Sohn, J. H.; Jung, M.; Lee, H.; Kim, S. H. J. Agric. Food Chem. 2012, 60, 9882.

[8] (a)Kim, S. G.; Kim, Y. M.; Khil, L. Y.; Jeon, S. D.; So, D. S.; Moon, C. H.; Moon, C. K. Arch. Pharm. Res. 1998, 21, 140.

(b) Hwang, G. S.; Kim, J. Y.; Chang, T. S.; Jeon, S. D.; So, D. S.; Moon, C. K. Arch. Pharm. Res. 1998, 21, 774

[9] Hikino, H.; Taguchi, T.; Fujimura, H.; Hiramatsu, Y. Planta Med. 1977, 31, 214
[10] Xu, H. X.; Lee, S. F. Phytother. Res. 2004, 18, 647.

[11] Moon, C. K.; Park, K. S.; Kim, S. G.; Won, H. S.; Chung, J. H. Drug Chem. Taxicol. 1992, 15, 81.

[12] Lee, Y. R.; Noh, E. M.; Han, J. H.; Kim, J. M.; Hwang, J. K.; Hwang, B. M.; Chung, E. Y.; Kim, B. S.; Lee, S. H.; Lee, S. J.; Kim, J. S. Eur. J. Pharmacol. 2012, 674, 80.

[13] Wang, X.; Zhao, H. X.; Bai, H. Acta Chin. Med. Pharmacol. 2013, 41, 164 (in Chinese). (王金金, 赵焕新, 白虹, 中医药学报, 2013, 41, 164.)

[14] (a) Zhao, Y. N.; Pan, Y.; Tao, J. L.; Xing, D. M.; Du, L. J. Pharmacology 2006, 76, 76

(b) Zhong, X.; Wu, B.; Pan, Y. J.; Zheng, S. Neoplasma 2009, 56, 387.

(c) Shen, J.; Zhang, H.; Lin, H.; Su, H.; Xing, D.; Du, L. Eur. J. Pharmacol. 2007, 558, 88.

(d) Ye, M.; Xie, W. D.; Lei, F.; Meng, Z.; Zhao, Y. N.; Su, H.; Du, L. J. Int. Immunopharmacol. 2006, 6, 426.

(e) Shen, J.; Yip, S.; Wang, Z. X.; Wang, W.; Xing, D. M.; Du, L. J. Eur. J. Pharmacol. 2008, 580, 366.

[15] Hill, R. A.; Parker, M. C. Nat. Prod. Rep. 2002, 19, iii.

[16] Heller, W.; Tamm, C. Progress in the Chemistry of Organic Natural Products, Vol. 40, Eds.: Herz, H.; Criesenbach, H.; Kirby, W, Springer-Verlag, Inc., New Work, 1980, p.105.

[17] Saitoh, T.; Sakashita, S.; Nakata, H.; Shimokawa, T.; Kinjo, J.; Yamahara, J.; Yamasakim, M.; Nohara, T. Chem. Pharm. Bull. 1986, 34, 2506.

[18] (a) Kirkiacharian, B. S. C. R. Acad. Ser. C 1972, 274, 2096. (b) Kirkiacharian, B. S.; Billet, D.; Durgeat, M.; Heitz, S.; Adjangba, M. K. Bull. Soc. Chim. Fr. 1975, 1770.

[19] Yen, C. T.; Kyoko, N. G.; Hwang, T. L.; Wu, P. C.; Morris-Natschke, S. L.; Lai, W. C.; Bastowd, K. F.; Chang, F. R.; Wu, Y. C.; Lee, K. H. Bioorg. Med. Chem. Lett. 2010, $20,1037$.

[20] (a) Davis, F. A.; Chen, B. C. J. Org. Chem. 1993, 58, 1751. (b) Davis, F. A.; Chen, B. C. Tetrahedron Lett. 1990, 31, 6823.

[21] Arnoldi, A.; Bassoli, A.; Borgonovo, G.; Merlini, L. J. Chem. Soc., Perkin Trans. 1 1995, 2447. 
[22] Ishii, H.; Koyama, H.; Hagiwara, K.; Miura, T.; Xue, G. G.; Hashimoto, Y.; Kitahara, G.; Aida, Y.; Suzuki, M. Bioorg. Med. Chem. Lett. 2012, 22, 1469.

[23] Javed, U.; Karim, M.; Jahng, K. C.; Park, J. G.; Jahng, Y. D. Tetrahedron: Asymmetry 2014, 25, 1270.

[24] Huang, Y. D.; Zhang, J. S.; Pettus, T. R. R. Org. Lett. 2005, 7, 5841.

[25] Lin, C. C.; Teng, T. M.; Tsai, C. C.; Liao, H. Y.; Liu, R. S. J. Am. Chem. Soc. 2008, 130, 16417.
[26] Pan, C. X.; Zeng, X. H.; Guan, Y. F.; Jiang, X. L.; Li, L.; Zhang, H. B. Synlett 2011, 425.

[27] Wang, X. Q.; Zhang, H. B.; Yang, X. D.; Zhao, J. F.; Pan, C. X. Chem. Commun. 2013, 49, 5405.

[28] Li, L. Q.; Li, M. M.; Wang, K.; Qin, H. B. Tetrahedron Lett. 2013, $54,6029$.

[29] Yadav, J. S.; Mishra, A. K.; Das, S. Tetrahedron 2014, 70, 7560.

[30] Jung, Y.; Kim, I. J. Org. Chem. 2015, 80, 2001.

(Qin, X.) 